\title{
BRONCHOGRAPHY IN PULMONARY TUBERCULOSIS
}

\author{
BY \\ PAUL FORGACS \\ From Kettlewell Hospital, Swanley, Kent
}

(RECEIVED FOR PUBLICATION FEBRUARY 22, 1955)

For 30 years after the introduction of bronchography by Sicard and Forestier (1922) iodized oil remained the only contrast medium in general use. During this period a large amount of information was published about the radiographic appearance of the bronchi in most varieties of chronic pulmonary disease. The literature dealing with bronchography in pulmonary tuberculosis is scanty by comparison. Early hopes that iodized oil might help to demonstrate tuberculous cavities met with disappointment, because the bronchi draining them are usually too narrow and tortuous to admit the contrast medium (Landau, 1925). Dilatation of the bronchi leading to tuberculous lesions was noted by Grill (1927), who also described stenosis of one of the main bronchi in one of his cases. The appearance of the bronchi in pulmonary tuberculosis was discussed in more detail by Leuret, Secousse, and Caussimon (1927), in an account based on 55 cases. They demonstrated fusiform and saccular dilatation of the bronchi, stenosis, and obstruction ; but, like others, they were rarely successful in outlining cavities. For some years after these early reports interest in bronchography of tuberculous patients was temporarily lost until the wider application of surgical treatment brought with it the need for more precise information about the topography of the infection. The practical value of bronchography in the localization of cavities was stressed for the first time by Neuhof (1934), while Potter and Pagliughi (1936) and Cabitt, Singer, and Graham (1936) found that the calibre of the bronchi under a thoracoplasty gave some indication of the success of the operation. The largest series of tuberculous patients studied by bronchography was reported in a series of papers by Dormer, Friedlander, and Wiles $(1944,1945)$, who laid emphasis on the more striking bronchial changes, especially dilatation, encountered amongst over 2,000 bronchograms. An attempt to estimate the incidence of bronchiectasis in tuberculous lesions was made by Murphy (1934), who noted it in $60 \%$ of his bronchograms, and by Boyer
(1946), who studied a series of 50 unselected cases and found dilatation in $85 \%$. The somewhat lower figure of $51.8 \%$ is given by Buckles, Potts, Davidson, and Neptune (1951) in their report on the bronchograms of 222 consecutive tuberculous patients.

As long as iodized oil remained the only contrast medium available for bronchography, the general reluctance to carry out this investigation on tuberculous patients was well founded. It was widely believed that a local tissue reaction to residues of oil or the general effects of the iodine liberated might aggravate the disease, and that the introduction of a large volume of liquid into the lung, irrespective of its chemical composition, might disseminate tubercle bacilli. The literature bearing on this subject was reviewed by Marchese, Klassen, and Curtis (1952), who also studied the effect of iodized oil in 49 of their own cases. They concluded that in stable or healing disease the method was safe, but in progressive pulmonary tuberculosis there was a risk of spreading the infection. Another objection to the use of iodized oil is its persistence. In a disease where progress is judged to a large extent by comparison of serial radiographs this disadvantage was serious enough by itself to make the investigation unpopular.

Several favourable reports have appeared in the last two years on the use of propyliodone ("dionosil") in pulmonary tuberculosis. They all agree that the risk of spreading the disease is negligible and that the clarity of subsequent radiographs is not impaired (McKechnie, 1953 ; Elphinstone, Iles, and Laidlaw, 1954 ; Holden, 1954). At Kettlewell Hospital aqueous propyliodone, introduced by crico-thyroid puncture, has been extensively used since 1952, without any complication more serious than a transient febrile reaction. Now that the safety of this procedure is established the time seems opportune to examine the first consecutive sets of 120 bronchograms in order to estimate the incidence of the bronchial changes and to assess the value of this investigation in pulmonary tuberculosis. 


\section{Selection of Cases}

A reliable estimate of the incidence of bronchographic abnormalities in tuberculosis could be based only on a series of unselected cases. The performance of such an uncomfortable investigation at random cannot be justified, and only those cases were therefore chosen where the bronchogram might have provided information of practical value. The grounds on which this selection was made were as follows. In one group of cases bronchial changes were expected because of lobar or segmental consolidation, rapid inflation of a cavity, or the presence of a large primary complex. In others bronchography was carried out before resection in order to study the extent of the disease, or to localize a small lesion accurately, and to provide a guide to the origin and distribution of the segmental bronchi. In a third group the purpose of the investigation was to exclude bronchial stenosis in patients about to undergo collapse therapy. Bronchograms were also taken occasionally both before and during pneumoperitoneum treatment to study the degree of relaxation of individual segments.

The extent and type of disease included in these groups are not readily amenable to classification. Of the 120 patients, 76 were men and 44 were women, between 17 and 67 years of age. They represent the population of a hospital treating all forms of pulmonary tuberculosis with the exception of advanced chronic disease. The bronchograms were usually taken within the first three months of treatment.

\section{ANALYSIS OF THE BRONCHOGRAMS}

Before considering the bronchographic findings in detail the limitations of this method of investigation should be appreciated. Stenosis and obstruction of peripheral bronchi, the study of which would be of great interest, are difficult to demonstrate. Appearances suggesting such changes may be due to incomplete filling; on the other hand a determined attempt to fill peripheral branches defeats its object by producing a network of overlapping shadows. Cavities can rarely be outlined owing to the small calibre of the draining bronchus; in the present series this was achieved in two cases only. Abnormal appearances regularly demonstrable by bronchography are confined to variations of calibre and deviation of the larger bronchi. Such changes may be of interest in themselves and may also provide a clue to the anatomical localization of lesions within their territory. When the outline of the bronchi is normal, the bronchial tree can be used as a grid of reference against which shadows in the lung may be accurately localized. The bronchograms also provide evidence of variation in the size of individual segments.
Normal Bronchograms.-In 50 patients the bronchograms were normal. While this was to be foreseen in patients with small and localized tuberculous lesions, it was surprising to find consolidation of a segment or a lobe resolve without leaving any distortion of the bronchi. Even where the lung still contained extensive and conspicuous lesions it was not unusual to find the bronchi leading to them perfectly normal. So much stress has been laid on bronchographic abnormalities in tuberculosis that their absence in nearly half of the cases in a series selected with a bias towards bronchial disease is worth noting.

Bronchial Dilatation.-This is the commonest of all bronchographic abnormalities seen in pulmonary tuberculosis. The picture is that familiar in mild chronic bronchiectasis, with beading of the larger bronchi and cylindrical widening of their branches. Gross bronchial dilatation comparable in degree with severe chronic bronchiectasis was not seen, even in segments or lobes destroyed by tuberculosis. This was unexpected in the light of previous reports which have laid emphasis on such changes (Dormer and others, 1945). In our series bronchial dilatation was found in 33 cases, and in all but eight it was slight.

DiverTiculosis.-Small diverticula, less than 1 $\mathrm{mm}$. wide and projecting $1-2 \mathrm{~mm}$. beyond the bronchial silhouette, were seen in 16 bronchograms (Fig. 1). They usually occurred in groups, giving a crenated outline to the bronchus, closely resembling diverticulosis of the colon on a miniature scale. The inferior profile of the left upper lobe bronchus and of the trunk of the lingula are the commonest sites of these diverticula. They may be encountered occasionally on the primary branches of the segmental bronchi, but do not occur more peripherally. Their appearance is identical with that of the diverticula described in chronic bronchitis (Stutz, 1948; Fischer, 1950 ; Simon and Galbraith, 1953). It is known that they represent dilated ducts of the bronchial mucous glands (Stutz, 1948 ; Duprez and Mampuys, 1953).

Bronchial Stenosis.-Stenosis of a lobar or segmental bronchus was demonstrated in two cases only. They were suffering from primary tuberculous infection of the lung supplied by the stenosed bronchus. In one of these the right upper lobe bronchus was narrowed to half its normal diameter and its anterior division tapered to a complete obstruction. A second bronchogram after eight months' treatment showed no change in the anterior segmental bronchus, but the localized stenosis of the lobar bronchus had disappeared (Fig. 2). The bronchograms of the other patient demonstrated stenosis in two places: in the left upper lobe bronchus, and in its superior division immediately beyond its origin. At both sites the bronchus was narrowed to half its normal calibre. This patient had already received six months' chemotherapy and the stenosis was therefore considered to be permanent.

The difficulty of demonstrating stenosis in peri- $\stackrel{\mathbb{D}}{\Omega}$ pheral bronchi has already been mentioned. Because 


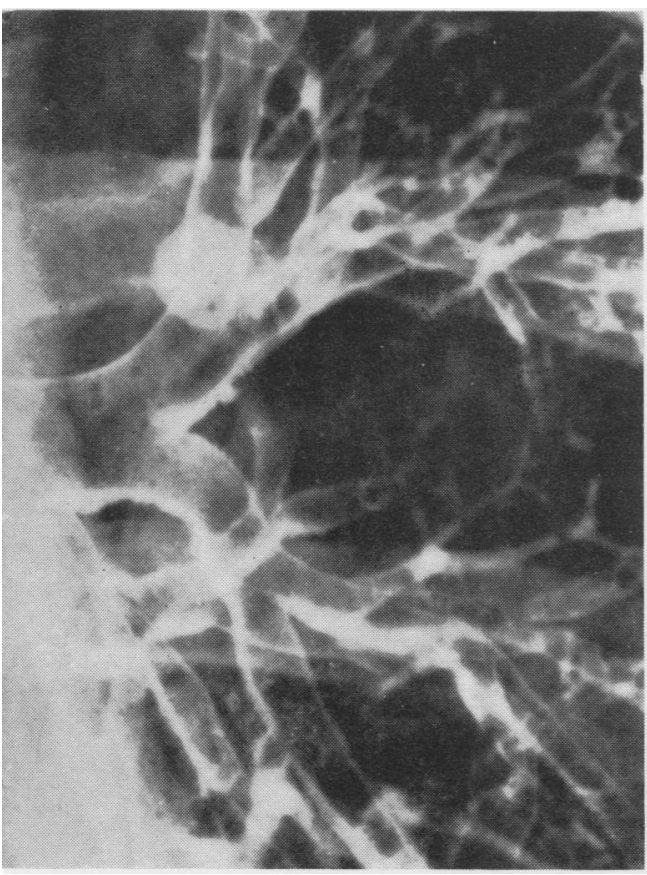

Fig. 1.-Diverticulosis of the left upper lobe bronchus and of its two primary divisions.

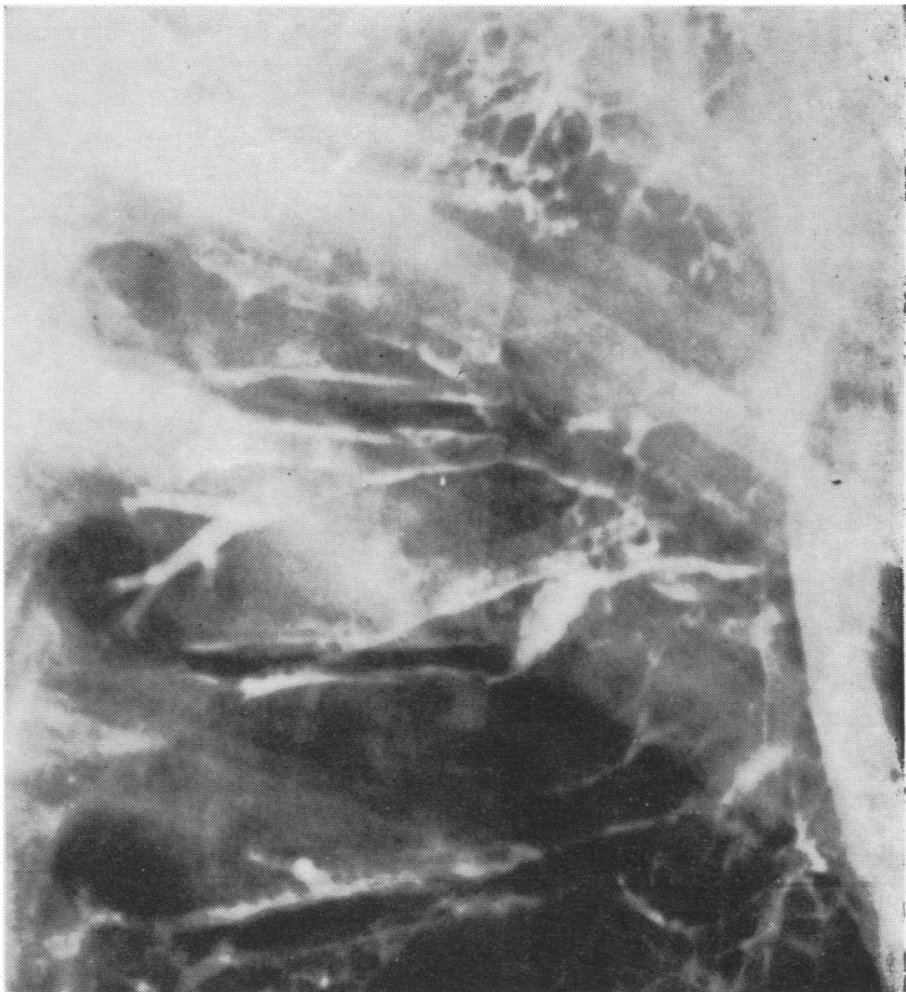

FIG. 3.-Characteristic tapering and irregularity of a bronchus draining a cavity in the apical segment of the right lower loke.

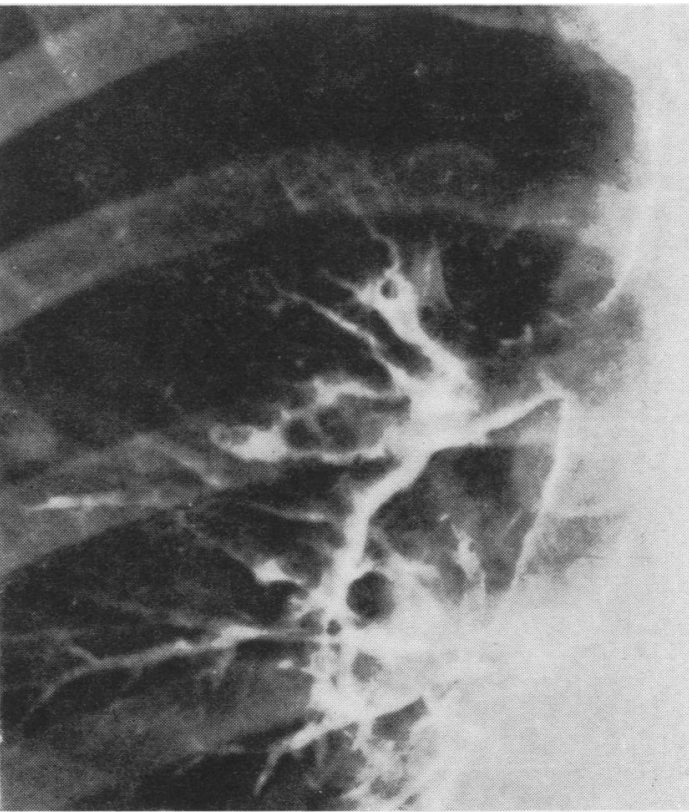

FiG. 2B.-After five months' chemotherapy the calitie of the right upper lobe bronchus is normal, but the obstruction of its anterior branch remains. 
of this no reliable estimate can be made of its incidence. Amongst the bronchograms in which peripheral filling was adequate and at the same time detail was not lost in the maze of superimposed shadows, stenosis of the bronchus draining a cavity was seen in three instances. The appearance of such bronchi is characteristic. The normally smooth silhouette of the bronchus becomes grossly irreguiar and tapers to a fine point over a distance of about $1 \mathrm{~cm}$. (Fig. 3).

Other ABnormalities.-Unexpected bronchiectasis of the lower lobe in the presence of abnormal shadows confined to the upper lobe was found in two patients. There was no indication in their past history, physical signs, and in the standard radiographs of disease of the lower lobe. In another patient collapse of the right middle lobe was discovered by bronchography.

\section{Practical Applications}

The conclusion reached from a review of 120 consecutive bronchograms of tuberculous patients is that the practical value of this investigation is limited. The information it provides is useful, but often obtainable by simpler methods. On the other hand, bronchography may occasionally demonstrate some unsuspected and important abnormality, and has therefore certain indications.

The most useful place of bronchography is amongst the preliminary investigations leading to major thoracic surgery. As a method of localizing tuberculous lesions to a segment or to its subdivisions it is helpful but not superior to lateral tomography. Both methods have their limitations: bronchography provides an excellent frame of reference, but the lesion to be localized is not so clearly shown as in tomograms, while the frame of reference provided by vessels, bronchi, and fissures in lateral tomograms is less precise, especially where segmental subdivisions are concerned. Bronchograms were found particularly valuable in localizing the disease to subdivisions of segments, and in deciding whether disease in the upper lobes was confined to the apical and posterior segments or extended into the anterior segment or into the apical segment of the lower lobe. Advance information about the configuration of the bronchial tree, especially in the anatomically variable left upper lobe, can also be useful at the time of the operation.

Similar conclusions about the value of bronchography before resection were reached by several authors. Gordon, Zinn, Brook, and Pratt (1951) compared their bronchograms with necropsy and resection specimens injected by radio-opaque material and found the bronchographic changes a useful guide to the localization of pulmonary lesions. They also pointed out that the bronchial changes in a lung covered by thickened pleura reflect the extent and severity of the disease and help to decide whether decortication is advisable. The advantages of bronchography in planning the extent of resections are stressed by Ibers, Vieten, and Willmann (1951), by Thurn (1954), and by Shaw, Collins, and MacNamara (1954).

The occasional discovery of unsuspected bronchiectasis of the lower lobe in the presence of tuberculosis would by itself justify the routine use of bronchography before resection. The chance finding of silent bronchiectasis in a healthy person may not be of immediate relevance, but in a tuberculous patient it demands investigation to see whether tubercle bacilli are established in the bronchiectatic lobe. The importance of this finding in the two patients reported was even greater, for in both resection of the upper lobe had been contemplated. Even where the difficult task of excluding a tuberculous infection in the bronchiectatic lobe can be accomplished in such circumstances, the danger of post-operative failure of expansion and of secondary infection remains. The choice lies between extending the resection to the bronchiectatic lobe and removing the lung, as was done in both these cases, or abandoning the idea of resection in favour of some more conservative line of treatment. This particular problem, with a case illustrating it, is mentioned by Rabinowitz and Harper (1951).

As a diagnostic aid in pulmonary tuberculosis bronchography is of limited value, since most of the bronchial changes discovered are not peculiar to this disease. The only exception is bronchial stenosis found at a distance from a lung shadow. This, being a feature of tuberculous bronchitis, may help to exclude other inflammatory or neoplastic conditions.

Where the diagnosis of pulmonary tuberculosis is already established, bronchography contributes little to a fuller understanding of the disease. The bronchi draining tuberculous cavities are usually too small to be studied by bronchography, and, although they may be demonstrated accidentally, more often they are lost in a network of superimposed shadows. The study of tuberculosis spreading along the submucosal lymphatic channels and of the lesions arising from this type of infection also proved unrewarding for the same reason. Cavities were outlined very rarely and in no instance was an unsuspected cavity demonstrated by the contrast medium.

The practical value of detecting dilated bronchi within a tuberculous lobe or segment is small. When the surrounding parenchyma is atelectatic, 
the dilation may be due entirely to the increased pressure gradient on either side of the bronchial wall, so that damage to the bronchus cannot be inferred. In most bronchograms, however, shrinking of the lung could not be held responsible for the bronchial dilation, which was therefore attributed to damage to the bronchial wall by the tuberculous infection. Active and healed tuberculous bronchitis could not be distinguished by the appearance of the dilated bronchi, since they were similar in untreated cases and in those which had received adequate chemotherapy. On bronchoscopy the segmental orifice leading to such dilated bronchi was in some patients congested and discharging pus ; in others the mucosa was normal, but mucus was oozing from the bronchus; in many cases, however, the bronchial orifice was normal. The conclusion to be drawn from these observations is that bronchial dilatation is as likely to be due to active as to healed tuberculous bronchitis, and that its presence merely reflects one aspect of the destruction wrought by the disease. It is rarely severe enough to be regarded as bronchiectasis in the clinical sense. It was never found to be responsible for the circular or oval translucencies in plain radiographs, which are so often attributed to it.

Diverticulosis of the bronchi may be a sequel of tuberculous bronchitis and can be interpreted as such when these bronchi lead to tuberculous parenchymal disease. It may also occur in other situations, where it may be the sequel of other forms of bronchitis. The reason for the high incidence of diverticula on the inferior profile of the left upper lobe bronchus and on the trunk of the lingular bronchus, common to chronic bronchitis and tuberculosis, is not known.

\section{COMPlications}

No serious complication occurred, and spread of tuberculosis, in particular, was never seen. The lungs were usually clear of contrast medium within 48 hours and always within a week, except on one occasion when propyliodone entered a cavity and persisted for 28 days.

Occasional fluctuations of temperature above $99^{\circ}$ F. $\left(37.2^{\circ}\right.$ C.) were common in these tuberculous patients, and a pyrexia exceeding $99.6^{\circ} \mathrm{F}$. $\left(37.6^{\circ}\right.$ C.) on the day following bronchography was therefore arbitrarily chosen as evidence of a reaction to propyliodone. It occurred in 34 patients, rarely lasted longer than 24 hours, and always settled in less than four days. The pyrexia was sometimes accompanied by malaise, headache, and pains in the limbs. Radiographs taken during this reaction did not show pulmonary consolidation, the white cell count and the sedimentation rate did not rise, and the urine remained normal. Amongst the 21 patients subjected to a second bronchography, usually after an interval of three months, this pyrexial reaction was observed on the first occasion alone in seven, on the second occasion alone in two, and both times in three. The amount of residual propyliodone in the lungs or the amount swallowed during the injection bore no relation to the incidence or severity of these symptoms. The cause of the reaction is probably a toxic effect of propyliodone absorbed from the lungs. It is evidently not due to infection or to sensitization by the contrast medium. It also occurs after bronchography in other diseases and cannot therefore be attributed to a tuberculin reaction.

\section{SUMmary}

A series of 120 bronchograms performed with aqueous propyliodone ("dionosil") in patients with pulmonary tuberculosis is reviewed. It is concluded that this procedure is safe and holds a modest place amongst other investigations as a preliminary to major surgical procedures, especially resection. It can be of considerable help in localizing the site of the disease with precision, and in demonstrating unsuspected bronchial abnormalities in radiologically intact parenchyma. Since no bronchographic feature peculiar to active tuberculosis was discovered, this investigation provides no indication of the state of healing of the disease.

\section{REFERENCES}

Boyer, L. B. (1946), Amer, Rev. Tuberc, 54 111.

Buckles, M. G., Potts, W. L., Davidson, H. B., and Neptune, W. B. (1951). Ibid., 64, 394.

Cabitt, H. L., Singer, J. J., and Graham, E. A. (1936). J. thorac. Surg., 5, 259.

Dormer, B. A., Friedlander, J., and Wiles, F. J. (1944). Amer. Rev. Tuberc., 50, 283

- - (1945). Ibid., 51, 62, 455, 519.

Duprez, A., and Mampuys, R. (1953). Thorax, 8, 141.

Elphinstone, N. C., Iles, K. C., and Laidlaw, E. F. (1954). Brit. J. Tuberc., 48, 33 .

Fischer, F. K. (1950). Fortschr. Röntgenstr., 72, 653.

Gordon, J., Zinn, B. W., Brook, R., and Pratt, P. C. (1951). J. thorac. Surg., 22, 109

Grill, C. (1927). Acta med. scand., 65, 300

Holden, W. S. (1954). Paper read at the Meeting of the British Tuberculosis Association, Oxford.

Ibers, G., Vieten, H., and Willmann, K. H. (1951). Fortschr. Röntgenstr., 74, 667.

Landau, W. (1925). Klin. Wschr., 4, 1861

Leuret, E., Secousse, H., and Caussimon, J. (1927). J. Méd. Bordeaux, 104, 215.

McKechnie, J. K. (1953). Tubercle, Lond., 34, 271.

Marchese, V., Klassen, K. P., and Curtis, G. M. (1952). Amer. Rev. Tuberc., 66, 699.

Murphy, J. E. (1934). Amer. J. Roentgenol., 31, 301.

Neuhof, H. (1934). Ibid., 31, 289.

Potter, B. P., and Pagliughi, J. (1936). Ibid., 36, 30.

Rabinowitz, P., and Harfer, I. S. H. (1951). Dis. Chest, 19, 66.

Shaw, K. M., Collins, D. M., and MacNamara, J. (1954). Amer. Rev. Tuberc., 70, 274.

Sicard, J. A., and Forestier, J. (1922). Bull. Soc. méd. Hôp. Paris 46, 463 .

Simon, G., and Galbraith, H. J. B. (1953). Lancet, 2, 850.

Stutz, E. (1948). Röntgenpraxis, 17, 91.

Thurn, P. (1954). Fortschr. Röntgenstr., 80, 198. 